\title{
DIFFERENTIAL IMPACT OF COVID-19 LOCKDOWN ON PHYSICAL ACTIVITY IN YOUNGER AND OLDER ADOLESCENTS - PROSPECTIVE STUDY
}

\author{
Marijana Geets Kesic ${ }^{1}$, Barbara Gilic ${ }^{1,2}$, Ivana Cerkez Zovko³, Patrik Drid ${ }^{4}$, Darinka Korovljev ${ }^{4}$ Damir Sekulic ${ }^{1}$ \\ ${ }^{1}$ University of Split, Split, Croatia \\ Faculty of Kinesiology \\ ${ }^{2}$ University of Zagreb, Zagreb, Croatia \\ Faculty of Kinesiology \\ ${ }^{3}$ University of Mostar, Mostar, Bosnia and Herzegovina \\ Faculty of Science and Education \\ ${ }^{4}$ University of Novi Sad, Novi Sad, Serbia \\ Faculty of Sport and Physical Education
}

\begin{abstract}
Background: Insufficient physical activity levels (PAL) during adolescence is a major public health concern, which is even more pronounced during the COVID-19 pandemic due to restricting movement opportunities. This study aimed to identify PAL changes and examine the age-specific determinants of PAL in younger and older adolescents during the COVID-19 lockdown. Material and Methods: This study included 859 high-school students from Bosnia and Herzegovina stratified into 2 age groups: younger $(\mathrm{N}=420,14-16$ years of age), and older adolescents $(\mathrm{N}=439,16-18$ years of age). Participants were tested over 2 testing waves: before the COVID-19 lockdown (January 2020) and during the COVID-19 lockdown (April 2020). Variables included PAL assessed by the Physical Activity Questionnaire for Adolescents, sports factors, and parental factors. Results: The PAL significantly declined as a result of lockdown measures in the total sample (from $2.76 \pm 0.79$ to $2.50 \pm 0.82$ ). Larger absolute and relative decline of PAL was evidenced in younger adolescents. Sport participation positively influenced PAL before lockdown, with no significant influence during the lockdown. Older adolescents whose mothers were better educated were less likely to be in high risk group with regard to a large decline of PAL as a result of COVID lockdown $(\mathrm{OR}=0.50,95 \% \mathrm{CI}: 0.21-0.84)$. Conclusions: Results of the study suggest that parental education influences health-related behaviors and that parental education is a protective factor against a decrease in PAL during the COVID-19 pandemic. Main educational agents (i.e., school and parents) should pay more attention to provide children and adolescents adequate information and develop their health literacy, which will hopefully positively impact children's PAL even in challenging situations similar to COVID-19 lockdown. Med Pr. 2021;72(6):633-43
\end{abstract}

Key words: pandemic, youth, sport participation, familial factors, physical activity, health literacy

Corresponding author: Damir Sekulic, University of Split, Faculty of Kinesiology, Teslina 6, 21000 Split, Croatia, e-mail: dado@kifst.hr Received: July 14, 2021, accepted: October 1, 2021

\section{INTRODUCTION}

Engaging in physical activity (PA) in adolescence is one of the main determinants of health status because it has been reported that PA can prevent many non-communicable diseases. Specifically, sufficient PA in adolescence is associated with a reduced risk of metabolic syndrome, obesity, coronary heart disease, and mental disorders [1]. However, it is a worrying fact that $81 \%$ of adolescents do not have adequate PA levels (PAL) globally, which is a major public health concern [2]. Specifically for Bosnia and Herzegovina, recent study recorded that children aged 14 years had 18\% lower PAL compared with 10 -year-old children, indicating alarming decline of PAL from childhood to adolescents, and the fact that that adolescents should be considered as vulnerable group for decreasing PAL [3]. Therefore, in order to be able to create adequate programs to increase PAL, numerous studies have examined which factors influence PAL among adolescents. In brief, previous studies identified various correlates of PA in adolescents including demographic factors (gender and age), behavioral factors (previous PA, sports participation, smoking and drinking alcohol), social factors (parental and friends support), and environmental factors (population density and living environment) [4]. Precisely, positive associations with PA were continuously confirmed for the male gender, urban living environment, sports participation, parental education, and parental and friends support [5].

Adolescence is the period in which the most pronounced decrease in PAL in life span occurs. In a review study involving children and adolescents aged 
10-19 years, it was recorded that the peak in PAL occurs at age 13, and PAL later begins to decrease by $7 \%$ annually [6]. Given that a decrease in PAL was recorded in pre-adolescents aged 10-14 years [3], due to dramatic changes in life habits (i.e., drop-out from sports, social influences, increased scholastic duties), an even greater decrease in PAL was expected in older adolescents. Supportively, a study by Miljanovic Damjanovic et al. [7] confirmed this hypothesis. Prospective studies in southeastern Europe additionally confirmed the decline in PAL in adolescents from the first to the second grade of high school (14-15 years of age) [8]; first to the second grade of high school (14-16 years of age) [9]; and third to the fourth grade of high school (16-18 years of age) [10]. Also, age-specific correlates of PAL in younger and older adolescents were observed. Specifically, positive changes in the PAL of younger adolescents were determined mainly by higher parental education [9], while parental conflict and the rural living environment determined a larger decline in PAL among older adolescents [11].

The proclamation of the COVID-19 pandemic in March 2020 has led to the introduction of social distancing measures and reduced movement opportunities. Precisely, stay-at-home restrictions, school closures, and cancellation of sports club practices led to a worldwide decrease in PAL among children and adolescents. Not surprisingly, studies in southeastern Europe recorded a decrease in PAL in adolescents during COVID-19 lockdown [12,13]. Apart from evidencing the changes in PAL, authors examined factors that have influenced changes in PAL among adolescents. Briefly, a greater decrease in PAL was observed in boys than in girls; the reduction in PAL was greater in urban than in rural adolescents, conflict with parents determined a larger decline in PAL, adolescents with better fitness status before pandemic had higher PAL during the pandemic [13], adolescents that participated in sports activities before the pandemic were more likely to have higher PAL during the pandemic [12], while adolescents who smoked cigarettes had lower PAL levels during the pandemic $[12,13]$.

From the previous literature review, it is evident that studies extensively examined changes in PAL as a result of the COVID-19 pandemic and factors which could influence such negative changes. However, practically all studies done so far examined adolescents of a relatively large age span (i.e., 14-18 years old, high-school students). More precisely, previous studies done in regular circumstances confirmed that younger adolescents had higher PAL than older adolescents and that different factors influence changes in PAL among older and younger adolescents [11]. Supportively, a study on Chinese adolescents noted that younger adolescents (12-15 years) had higher PAL than older adolescents (15-18 years) during the COVID-19 pandemic [14]. Finally, it seems that trends of higher PAL in younger age groups is characteristic even for lockdown period, since recent study evidenced that Canadian children (5-11 years) were more physically active than youth (12-17 years) during lockdown [15].

Collectively, there is a lack of prospective data about changes in PAL during the COVID-19 pandemic in different age groups of adolescents (i.e., younger adolescents aged $14-16$ years vs. older adolescents aged $16-18$ years). Also, to the best of the authors' knowledge, no study examined factors that may influence changes in PAL, which occurred during the COVID-19 pandemic, specifically in younger vs. older adolescents. Therefore, the aim of this study was to examine the changes in PAL which occurred as a result of the COVID-19 pandemic, and to examine the age-specific socio-demographic-, familial-, and sport-factors as correlates of PAL among younger and older adolescents during the COVID-19 lockdown. The authors hypothesized that observed predictors will positively influence PAL of studied adolescents before and during the COVID-19 lockdown, irrespective of participants' age. This data is vital in developing targeted interventions in terms of improving PAL during COVID-19 lockdown and similar crises in different age groups.

\section{MATERIAL AND METHODS}

\section{Participants and design of the study}

This research included 859 students attending high schools from several regions in Bosnia and Herzegovina (Tuzla, Herzegovina-Neretva, and West Herzegovina Canton). For the purpose of this study, the total sample was stratified into two subsamples: younger adolescents $(\mathrm{N}=420,14-16$ years of age at study baseline) and older adolescents $(\mathrm{N}=439,16-18$ years of age at study baseline). Before the study initiation, students regularly attended physical education classes at least twice a week. The response rate (based on responses at follow-up please see later for details on design), was $86 \%$. All participants gave their informed consent, and a parent or a legal guardian gave their approval for those participants younger than 18 years. The investigation was approved by the Ethical Board of the University of Split, Faculty of Kinesiology, Croatia (EBO: 2181-205-05-02-05-14-005).

This prospective study was commenced over 2 testing waves: 1) baseline, representing the measurement 

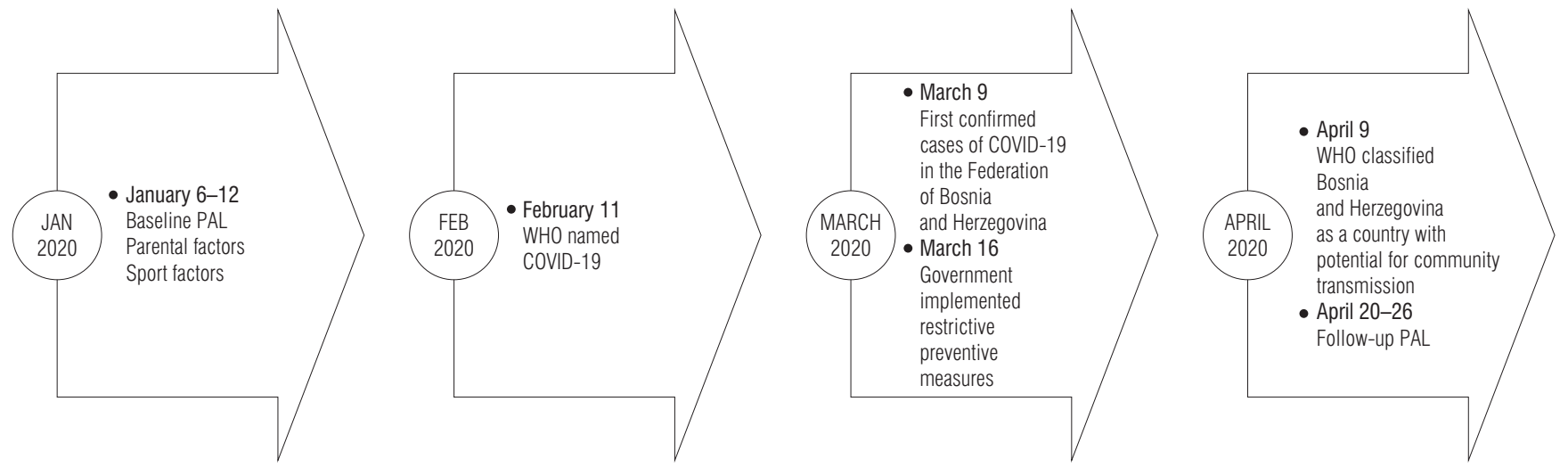

PAL - physical activity levels.

Figure 1. Timeline of the study done in Bosnia and Herzegovina and most important dates in first half of 2020

before implementation of the social distancing measures due to the COVID-19 pandemic (January 2020), and 2) follow-up, representing the measurement during the period when social distancing measures were implemented (i.e., COVID-19 lockdown; late April 2020). Baseline measurement included socio-demographic factors, parental/familial factors, sport factors, and baseline PAL. Follow-up measurement was conducted during the lockdown and included only follow-up PAL. In order to preserve participants' anonymity and collect more honest and precise answers in the questionnaire, students were asked to create their personal code and use it at baseline and follow-up measurement. The design of the study and detailed information about the study period are presented in Figure 1.

\section{Variables}

This study included socio-demographic factors (age, gender), parental/familial factors, sport factors (independent variables), and PAL (dependent variable).

The Physical Activity Questionnaire for Adolescents (PAQ-A) was used for assessing baseline and follow-up PAL [16]. PAQ-A has been frequently used for assessing PAL at similar samples of adolescents and has displayed appropriate validity and reliability $[7,10]$. It is a self-administered questionnaire that assesses the frequency of PA during the last 7 days. It consists of 9 items, first 8 assess PA during spare time, during physical education classes, after school, during evenings, and during the weekend, and are scaled $1-5$, representing low and high PA, respectively. The ninth item questions whether participants were injured or ill and is not included in the final score, which is calculated as the arithmetic mean of the first 8 items. In this study, the authors observed crude results of PAL at baseline $\left(\mathrm{PAL}_{\mathrm{BL}}\right)$, and PAL at follow-up $\left(\mathrm{PAL}_{\mathrm{FU}}\right)$. Next, crude PAL was also observed as the binomial variable with 2 categories: results; insufficient/inadequate vs. sufficient/adequate PAL (scores $\leq 2.73$ and $>2.73$, respectively), as suggested previously [17]. Further, to quantify the changes in $\mathrm{PAL}_{\mathrm{BL}}$ and $\mathrm{PAL}_{\mathrm{FU}}$, the authors calculated crude numerical difference between these 2 values $\left(\mathrm{PAL}_{\Delta}=\mathrm{PAL}_{\mathrm{BL}}-\mathrm{PAL}_{\mathrm{FU}}\right)$. Next, the authors calculated relative changes in PAL between baseline and follow-up (in \%) using the calculation: $\mathrm{PAL}_{\Delta \%}=\left(\mathrm{PAL}_{\mathrm{BL}}-\mathrm{PAL}_{\mathrm{FU}}\right) / \mathrm{PAL}_{\mathrm{BL}} \times 100$. For the purpose of later statistical calculations, participants were ordered according to their $\mathrm{PAL}_{\Delta \%}$, and then grouped into 2 groups (0-50 percentiles, and above). The participants with a greater relative decline of PAL (ordered $>50$ percentiles) were evidenced as "high risk group", while those placed in the first 50 percentiles were evidenced as "low risk group". Such dichotomization allowed the authors to calculate logistic regression for $\mathrm{PAL}_{\Delta \%}$ as a binomial criterion (please see later for details).

Parental/familial factors included the following variables: self-reported socioeconomic status (below average-average-above average); maternal and paternal education level (university degree-college degree-high school-elementary school); conflict with parents (never-rarely-from time to time-regularly/frequently); parental absence from home (never-rarely-from time to time-regularly/frequently); and parental/familial care (very poor care-low care-my parents/family care about me-my parents/family care about me a lot).

Sports factors were assessed by questions on participation in individual or team sports (with answers: never been involved-quit-currently involved), best results in competitions (with answers on a 4-point scale ranging from "never competed" to "international competitions"), and years of involvement in sports (with answers: never involved-less than one year-two to five years-more than five years). 


\section{Statistics}

Normality of the distribution was checked by Kolmogorov Smirnov's test, and descriptive statistics included means and standard deviations (for numerical variables) and frequencies and percentages (for ordinal and nominal variables).

Differences between groups were evidenced by t-test for independent samples (for quantitative variables), and Chi square $\left(\chi^{2}\right)$. T-test for dependent samples was used to identify the changes in PAL between baseline (pre-pandemic period) and follow-up (lockdown period).

Logistic regression (with Odds Ratio - OR, and Confidence Interval - CI reported) was applied to evidence the association between predictors and categorized PAL (insufficient PAL - coded as "1" vs. sufficient PAL - coded as "2") at baseline and follow-up.

Also, in order to identify the association which may exist between predictors and changes that occurred in PAL, the authors additionally calculated logistic regression with dichotomized $\mathrm{PAL}_{\Delta \%}$ as a criterion (low-risk group - coded as "1" vs. high-risk group - coded as “2").

All analyses were stratified for age groups (e.g., younger vs. older adolescents).

A p-value of $95 \%$ was applied, and the Statistica ver. 13.5 statistical package (Tibco Inc., CA) was used for all calculations.

\section{RESULTS}

Age groups significantly differed in self-perception of socioeconomic status, with somewhat better familial socioeconomic status among older adolescents $\left(\chi^{2}=9.05\right.$, $\mathrm{p}<0.01)$. Younger adolescents were more involved in individual sports $\left(\chi^{2}=25.76, \mathrm{p}<0.01\right)$, and team sports $\left(\chi^{2}=35.71, \mathrm{p}<0.01\right)$ than older adolescents, while sport achievement was higher in older adolescents $\left(\chi^{2}=23.76\right.$, $\mathrm{p}<0.01$ ) at baseline testing (Table 1).

At baseline, $55 \%$ of younger and $43 \%$ of older adolescents had sufficient PAL, while only $35 \%$ of younger and $28 \%$ of older adolescents had sufficient PAL at follow-up (during lockdown). Observing in crude PAQ-A results, the PAL significantly declined as a result of lockdown measures in the total sample (from $2.76 \pm 0.79$ to $2.50 \pm 0.82, \mathrm{p}<0.001)$, and separately in older adolescents (from $2.52 \pm 0.68$ to $2.31 \pm 0.74, \mathrm{p}<0.01$ ), and in younger adolescents (from $3.02 \pm 0.82$ to $2.71 \pm 0.85$ ), $\mathrm{p}<0.001$. Younger adolescents had higher PAL than their older peers at baseline (before lockdown) and follow-up (during lockdown) (Figure 2). Significant

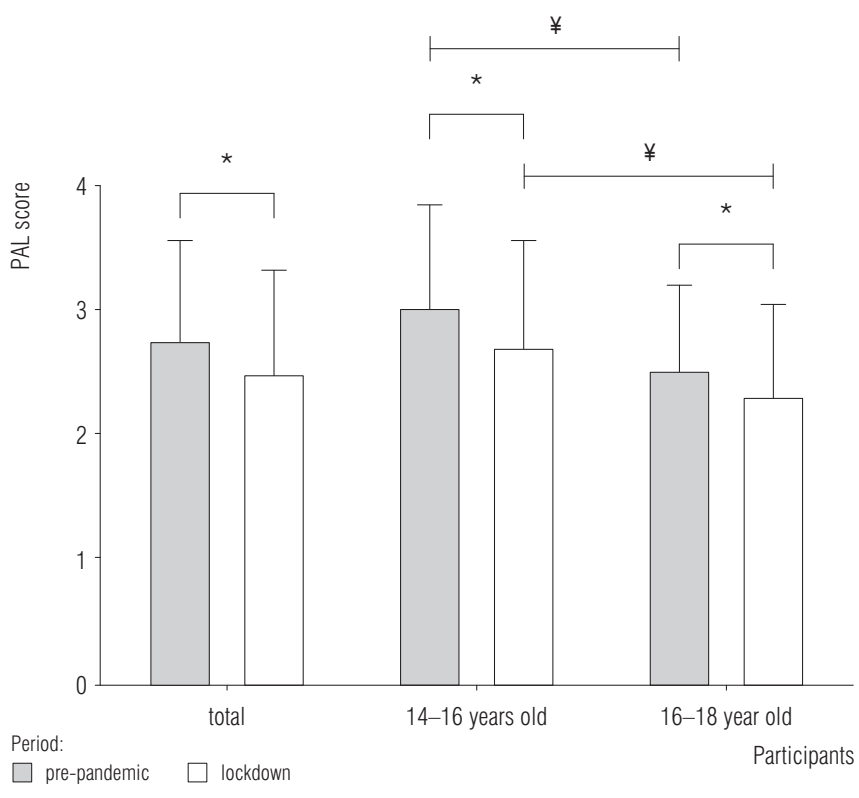

Significant $(\mathrm{p}<0.001)$ differences between $(¥)$ and within groups $\left(^{*}\right)$.

Figure 2. Descriptive statistics, changes and t-test differences in physical activity levels (PAL) before and during the COVID-19 lockdown in Bosnia and Herzegovina in 2020

differences between age groups were evidenced in $\mathrm{PAL}_{\Delta}$ ( $\mathrm{t}$-test $=1.98, \mathrm{p}<0.01)$, with larger decrease in younger adolescents, than in older adolescents $(0.31 \pm 0.80$ and $0.21 \pm 0.68$ for younger and older adolescents, respectively), with significant difference in $\mathrm{PAL}_{\Delta \%}$ between age groups $(\mathrm{t}$-test $=1.76, \mathrm{p}=0.04)$.

Table 2 presents results of logistic regression analysis for dichotomized PAL at baseline and follow-up as criterion variable (insufficient vs. sufficient PAL). In brief, for the pre-pandemic period, sufficient PAL was more likely to be achieved in males (for both age groups), in adolescents whose fathers were better educated (only among younger adolescents), in adolescents whose mothers were better educated (for both age groups), in adolescents who participated in individual sports (for both age groups), and those who participated in team sports (for both age groups). Sufficient PAL during the lockdown period was more likely to be achieved in males (for younger adolescents), adolescents whose mothers were better educated (for the older group), adolescents who declared better parental care (for the older group), those who participated in individual sports (for younger age group), and those who achieved better sport success (for younger age group).

Figure 3 presents associations between studied predictors and $\mathrm{PAL}_{\Delta \%}$, separately for younger and older adolescents. The only significant predictor was the level of maternal education. More precisely, older adolescents whose 
Table 1. Descriptive statistics and differences between age groups of adolescents from Bosnia and Herzegovina in early 2020

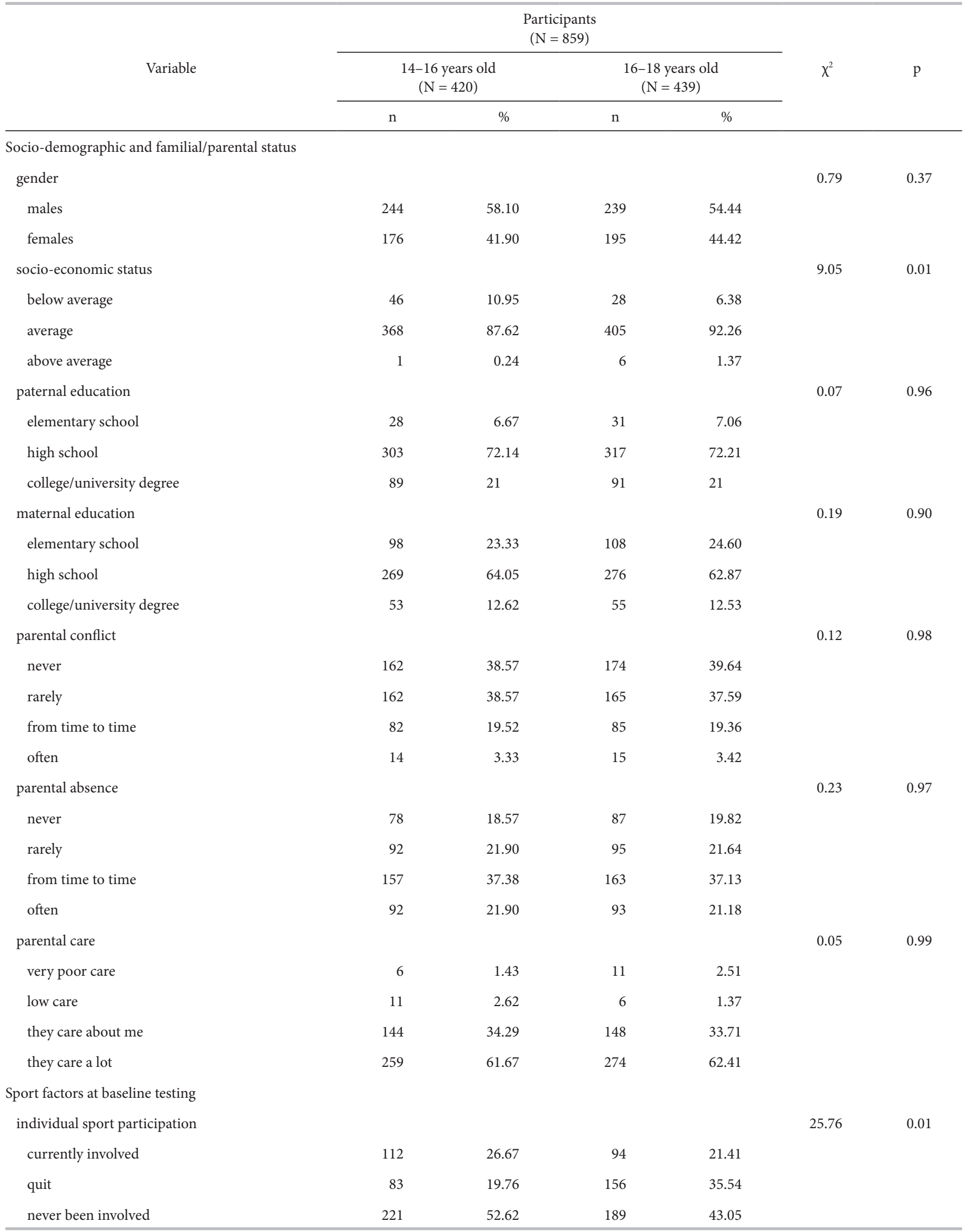


Table 1. Descriptive statistics and differences between age groups of adolescents from Bosnia and Herzegovina in early 2020 - cont.

\begin{tabular}{|c|c|c|c|c|c|c|}
\hline \multirow{3}{*}{ Variable } & \multicolumn{4}{|c|}{$\begin{array}{l}\text { Participants } \\
(\mathrm{N}=859)\end{array}$} & \multirow{3}{*}{$\chi^{2}$} & \multirow{3}{*}{$\mathrm{p}$} \\
\hline & \multicolumn{2}{|c|}{$\begin{array}{c}14-16 \text { years old } \\
(\mathrm{N}=420)\end{array}$} & \multicolumn{2}{|c|}{$\begin{array}{l}16-18 \text { years old } \\
(\mathrm{N}=439)\end{array}$} & & \\
\hline & $\mathrm{n}$ & $\%$ & $\mathrm{n}$ & $\%$ & & \\
\hline \multicolumn{7}{|c|}{ Sport factors at baseline testing - cont. } \\
\hline team sport participation & & & & & 35.71 & 0.01 \\
\hline currently involved & 168 & 40.00 & 102 & 23.23 & & \\
\hline quit & 106 & 25.24 & 181 & 41.23 & & \\
\hline never been involved & 144 & 34.29 & 156 & 35.54 & & \\
\hline sport experience & & & & & 7.70 & 0.07 \\
\hline never been involved & 109 & 25.95 & 106 & 24.15 & & \\
\hline$<1$ year & 67 & 15.95 & 101 & 23.01 & & \\
\hline $2-5$ years & 150 & 35.71 & 129 & 29.38 & & \\
\hline$>5$ years & 89 & 21.19 & 103 & 23.46 & & \\
\hline sport achievement & & & & & 23.76 & 0.01 \\
\hline never competed & 193 & 45.95 & 215 & 48.97 & & \\
\hline lower rank competition & 149 & 35.48 & 190 & 43.28 & & \\
\hline regional competition & 50 & 11.90 & 25 & 5.69 & & \\
\hline national/international level & 21 & 5.00 & 5 & 1.14 & & \\
\hline
\end{tabular}

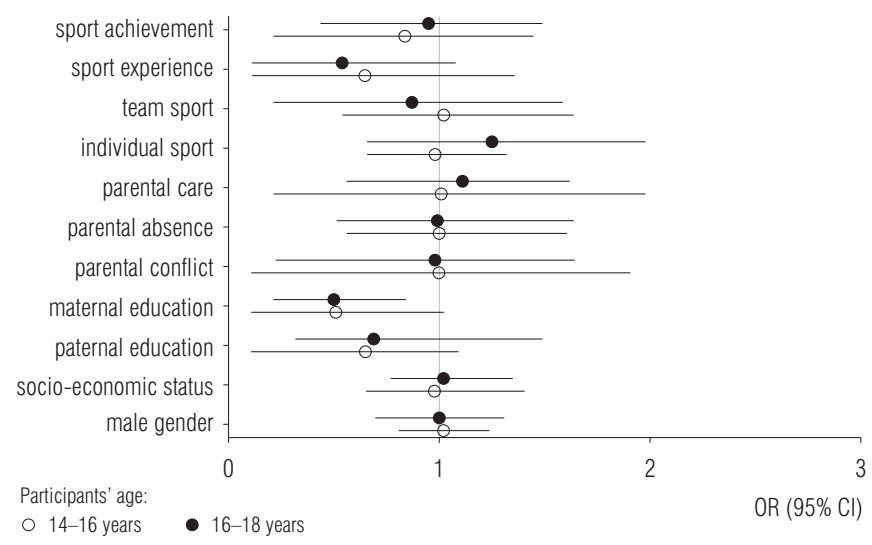

Figure 3. Associations between independent variables and relative changes in physical activity levels $\left(\mathrm{PAL}_{\Delta \%}\right)$ in younger and older adolescents (with group of higher risk for large decrease of PAL as reference category) from Bosnia and Herzegovina in 2020

mothers were better educated were less likely to be in "highrisk group" with regard to a large decline of PAL as a result of COVID lockdown (OR = 0.50, 95\% CI: 0.21-0.84).

\section{DISCUSSION}

The main aim of this study was to investigate the impact of mainly scholastic factors on PAL, and changes in PAL during the COVID-19 pandemic in younger and older adolescents. Apart from the expected decrease of PAL as a result of COVID-19 lockdown, there are several most important findings of this study. First, a larger absolute and relative decrease of PAL was evidenced in younger adolescents. Second, maternal education was a stronger factor of influence on PAL in older (16-18 years) adolescents. Third, a lower decrease of PAL was evidenced in older adolescents whose parents were better educated. Therefore, the authors' initial hypothesis might be partially accepted.

\section{PAL changes in younger and older adolescents}

The total sample of participants declined their PAL. This is in accordance with previous studies conducted on adolescents globally, including the adolescents from Bosnia and Herzegovina and neighboring countries $[12-14,18]$. However, the novel finding of this study is that younger adolescents had higher PAL than older adolescents in the pre-COVID period, with no significant difference between age groups in PAL for the pandemic period. Almost certainly, the lack of differences in PAL between age groups during COVID-19 lockdown can be attributed to a larger decline in PAL due to COVID-19 
lockdown in younger adolescents. On the other hand, both findings (higher pre-pandemic PAL, and greater decrease of PAL in younger adolescents) may be associated with participation in sports.

In brief, the results of the study confirmed higher involvement in sports among younger adolescents (please see Results for details). The participation in sports contributes to a large part of the total PAL in children and adolescents [19]. It was repeatedly reported that adolescents involved in organized sports activities have significantly higher PAL than their peers who do not participate in sports [20]. Further, it is known that there is the largest sport drop-out rate at the age of 14-15 years [21]. Evidenced differences in sports participation between younger and older adolescents are likely to occur due to increased commitments, lack of free time, focus on academic achievement [6], socialization with friends in sedentary activities, and employment in older adolescents [20].

It is also important to note that the high-school period is known for dropping out of sport as adolescents are mainly oriented towards competitive success in sports. At the same time, adolescents are not sufficiently educated about the importance of PA and its' positive impact on health [22]. Supportively, a study on adolescents from Portugal suggested that the degree of participation in sports is related to competitive sport achievement, which limits participation only to students who have better physical predispositions and who are talented [23].
Meanwhile, another Portuguese study highlighted insufficient education about the importance of the impact of $\mathrm{PA}$ on health as an even more likely cause of giving up sports [24].

Collectively, older adolescents in this study have lower PAL most likely as a consequence of dropping out from organized sports, compared to younger adolescents who are not yet at the point of the decision to continue or stop sports participation. Putting it altogether, it is absolutely logical that younger adolescents reduced their PAL to a greater extent during the COVID-19 lockdown simply because they did not have the opportunity to participate in sports activities during follow-up (i.e., sports clubs, facilities, and organized recreational activities were closed and banned during the lockdown period). Finally, as younger adolescents generally had higher PALs than older adolescents in the pre-lockdown period, it is understandable that their PAL decreased to a larger extent than the PAL of their older peers.

\section{Parental factors and PAL before and during lockdown}

Parental education is known to be an important factor of influence on children's PAL [25], and the authors' results of the study support such considerations. The association can be explained through the background of general parents' influence on the behavioral patterns of their children. Precisely, parents with their attitudes,

Table 2. Associations between independent variables and physical activity levels (sufficient physical activity - reference group) before and during COVID-19 lockdown in younger and older adolescents from Bosnia and Herzegovina in early 2020

\begin{tabular}{|c|c|c|c|c|c|c|c|c|}
\hline \multirow{3}{*}{ Variable } & \multicolumn{4}{|c|}{ Pre-pandemic period } & \multicolumn{4}{|c|}{ Lockdown period } \\
\hline & \multicolumn{2}{|c|}{$\begin{array}{l}14-16 \text { year old participants } \\
\qquad(\mathrm{N}=420)\end{array}$} & \multicolumn{2}{|c|}{$\begin{array}{l}16-18 \text { year old participants } \\
\qquad(\mathrm{N}=439)\end{array}$} & \multicolumn{2}{|c|}{$\begin{array}{l}14-16 \text { year old participants } \\
\qquad(\mathrm{N}=420)\end{array}$} & \multicolumn{2}{|c|}{$\begin{array}{l}16-18 \text { year old participants } \\
\qquad(\mathrm{N}=439)\end{array}$} \\
\hline & OR & $95 \% \mathrm{CI}$ & OR & $95 \% \mathrm{CI}$ & OR & $95 \% \mathrm{CI}$ & OR & $95 \% \mathrm{CI}$ \\
\hline Male gender & 1.25 & $1.09-1.4$ & 1.2 & $1.01-1.44$ & 1.11 & $1-1.21$ & 1.09 & $0.88-1.38$ \\
\hline Socio-economic status & 0.99 & $0.5-1.5$ & 0.98 & $0.6-1.42$ & 0.99 & $0.44-1.6$ & 1.02 & $0.55-1.59$ \\
\hline Paternal education & 1.61 & $1.25-1.88$ & 1.44 & $0.98-1.88$ & 1.32 & $0.8-1.76$ & 1.3 & $0.45-2.32$ \\
\hline Maternal education & 1.55 & $1.21-1.86$ & 1.74 & $1.44-2.05$ & 1.32 & $0.88-1.71$ & 1.66 & $1.11-2.2$ \\
\hline Parental conflict & 0.78 & $0.44-1.11$ & 0.8 & $0.5-1.1$ & 0.88 & $0.45-1.11$ & 0.81 & $0.32-1.44$ \\
\hline Parental absence & 0.75 & $0.44-1.09$ & 0.8 & $0.61-1.02$ & 0.91 & $0.77-1.33$ & 0.95 & $0.46-1.41$ \\
\hline Parental care & 0.89 & $0.65-1.21$ & 0.9 & $0.7-1.11$ & 1.21 & $0.98-1.56$ & 1.31 & $1.02-1.61$ \\
\hline Individual sports & 2.09 & $1.5-2.61$ & 1.66 & $1.33-1.99$ & 1.45 & $1.03-1.88$ & 1.21 & $0.81-1.65$ \\
\hline Team sports & 2.21 & $1.95-2.55$ & 2 & $1.75-2.28$ & 1.28 & $0.71-1.76$ & 1.11 & $0.45-1.88$ \\
\hline Sport experience & 1.92 & $1.71-2.21$ & 1.44 & $0.97-1.98$ & 1.09 & $0.51-1.65$ & 1 & $0.87-1.36$ \\
\hline Sport achievement & 1.84 & $1.11-2.5$ & 1.22 & $0.74-1.75$ & 1.54 & $1.11-1.91$ & 1.21 & $0.66-1.89$ \\
\hline
\end{tabular}


values, and knowledge guide their children to form their own behavioral patterns, including participating in any form of PA [25]. Parents are acting as role models to their children, meaning that parents who are physically active and who promote PA with their behavior positively influence PA habits of their children.

This phenomenon occurs according to the theory that children imitate parents and their habits, which means that children are likely to adopt PA habits as well. Besides the importance of role modeling and parental encouragement, attitudes and tangible support are considered as important determinants of parental influence on children's PAL. Thus, parents with higher educational levels may better perceive the health-related benefits of PA, which will provoke greater parental support and encouragement for PA and influence the overall formation of healthy habits of their children.

Previous studies noted somewhat different influences of maternal and paternal education on their children's PAL. Specifically, fathers are more likely to act as role models for PAL and sports activities compared with mothers. Furthermore, fathers more frequently engage in PA and are actively involved in PA with their children in terms of co-physical activities [26]. On the other hand, mothers have a great role in their children's educational achievement, which includes health-related education. Supportively, a study on Nigerian children noted that mothers with higher education levels had better knowledge of PA [27]. It is assumed that better educated and informed mothers possess higher health literacy skills that they transmit to their children and create a positive influence on making healthier decisions and encouraging children to participate in PA and other health behaviors [28].

Therefore, the authors' finding that maternal education positively influences PAL during the COVID-19 lockdown in older adolescents could be explained in the context of the situation and period when the study was conducted. Namely, during the pre-pandemic period, adolescents regularly participated in sports, and consequently, fathers probably had a stronger influence on their PAL. Meanwhile, during the COVID-19 lockdown, adolescents were required to stay at home due to movement restrictions (school and sports facilities closure). Therefore, they were probably more exposed to the control and influence of mothers.

The study recorded that the influence of maternal education on PAL in children was more pronounced in older adolescents. A recent study evidenced that younger adolescents (14-16 years of age) with bettereducated mothers and fathers had a higher likelihood of having sufficient PAL in regular circumstances [9]. However, a study on older adolescents (16-18 years of age) evidenced that paternal education was correlated with adolescents' PAL aged 16 years old, but at the age of 17 years, the correlation started to weaken [11]. Moreover, prospective studies on older adolescents (16-18 years old) reported that adolescents with more educated mothers are more likely to incline PAL during late adolescence $[7,10]$. This altogether indicates that the influence of paternal education on their children's PAL weakens during late adolescence, while the level of maternal education remains the significant influential factor on PAL even in late adolescence.

This is one of the first studies to prove that parental education acts as a preventive factor against a decrease of PAL during a COVID-19 lockdown. The results of this study can be supported by recent research on Spanish children, which recorded a more evident PAL decline during the COVID-19 lockdown in children (10-14 years) whose mothers were less-educated [29]. However, the authors' results of this study extend the previous knowledge regarding factors of influence on changes in PAL during the COVID-19 lockdown, since the authors investigated older children/adolescents (1618 years of age) than authors' Spanish colleagues, and showed a significant influence of both maternal and paternal educational levels on changes in PAL.

The fact that maternal education prevents the decline of PAL leads the authors to conclude that children of better-educated mothers are physically active more because of health-related issues and not because of involvement in competitive sports. Also, it is reasonable to hypothesize that older adolescents had better "health literacy" and that they knew how important it was to maintain appropriate PAL even during the lockdown. If the authors define health literacy as "the ability to selectively access and analyze information and to take action to promote personal and someone else's health behaviors" [30], the preventive effect of better maternal education on the decrease of PAL during lockdown is understandable.

Supportively, previous studies noted that adolescents who had better fitness status and were involved in sports before the pandemic efficiently maintained PAL during the pandemic $[12,13]$. The authors explained this finding with the term "physical literacy" precisely, with the assumption that adolescents of advanced fitness status have sufficient knowledge and motivation to create exercise programs themselves even with limited equipment and space during the lockdown. Health literacy 
and physical literacy, although not the same, are considered to be lifelong health-promoting behaviors [30]. Therefore, it is likely that adolescents whose mothers were better educated had better health literacy, recognized the importance of PA for their health, and strived to maintain PAL even during COVID-19 lockdown.

Considering all the above mentioned, it is not surprising that the authors have found a stronger influence of maternal education on PAL in older than in younger adolescents. It can be assumed that mothers influence adolescent habit formation and that older adolescents will more likely recognize and use it. On the other hand, younger adolescents are still in the process of forming their habits and may not yet be able to identify and choose what is good for them in terms of healthy behavior. In other words, younger children are more likely to participate in PA because of fun and parental supervision, while older children are more likely to participate in PA because they know what the health benefits of the PA are [10].

\section{Limitations and strengths}

Most of the variables were self-reported, which could be considered as the main limitation of the study. Precisely, students might answer some questions not completely honestly and provide more socially desirable answers. However, participants filled in questionnaires anonymously (for the purpose of repeated testing they use confidential codes); therefore, leaning towards socially desirable answers was less likely. In this research results on PAQ-A were clustered into 2 groups, which undoubtedly allocated participants with similar results into opposed groups. In the future studies division into three groups is therefore suggested.

This is one of the first studies investigating the changes and correlates of PAL during the COVID-19 lockdown in relation to age groups of adolescents (younger and older adolescents). Therefore, this study expands the knowledge on physical activity in adolescents during the COVID-19 pandemic and other similar situations and creates the background for future research. Additionally, this study used a measurement instrument (PAQ-A) which is widely used in studies on similar participants; therefore, the results of this study could be compared with other similar studies.

\section{CONCLUSIONS}

The greater decline in PAL during the COVID-19 lockdown was evidenced in younger adolescents compared to older adolescents, which was attributed to higher pre-pandemic PAL of younger adolescents and younger adolescents' higher engagement in sports in the pre-pandemic period.

This study's results can be considered great value for creating public-health policies for improving PAL in adolescents in regular circumstances and in situations similar to COVID-19 lockdown. Specifically, in order to achieve proper PAL, younger adolescents should be encouraged to continue participating in sports with an emphasis on the health benefits of PA and not on competitive achievements.

Schools or sports clubs should promote sports as health-enhancing behavior and teach children to participate in sports for their health regardless of competitive achievement. It will have direct positive repercussions on PAL in situations similar to COVID-19 lockdown when circumstances will limit regular sports participation. In such situations, adolescents with better health literacy will be able to recognize the importance of PA and will try to maintain sufficient PAL. Further studies are needed in order to:

evaluate a specific insight into various components of PAL during lockdown (i.e., physical education, sports, and free time),

overview the changes which occur in each component, and

investigate the factors of influence on changes in each component.

\section{ACKNOWLEDGMENTS}

Authors are particularly grateful to all children who voluntary participated in the study. Special thanks goes to school authorities who approved the investigation and helped in research

\section{REFERENCES}

1. Pavičić Žeželj S, Kenđel Jovanović G, Krešić G. The association between the Mediterranean diet and high physical activity among the working population in Croatia. Med Pr. 2019;70(2):169-176. https://doi.org/10.13075/mp.5893. 00773.

2. Guthold R, Stevens GA, Riley LM, Bull FC. Global trends in insufficient physical activity among adolescents: a pooled analysis of 298 population-based surveys with 1.6 million participants. Lancet Child Adolesc Health. 2020;4(1):23-35. https://doi.org/10.1016/s2352-4642(19)30323-2.

3. Pojskic H, Eslami B. Relationship Between Obesity, Physical Activity, and Cardiorespiratory Fitness Levels in Children 
and Adolescents in Bosnia and Herzegovina: An Analysis of Gender Differences. Front Physiol. 2018;9:1734. https://doi.org/10.3389/fphys.2018.01734.

4. Sallis JF, Prochaska JJ, Taylor WC. A review of correlates of physical activity of children and adolescents. Med Sci Sports Exerc. 2000;32(5):963-975. https://doi.org/10.1097/ 00005768-200005000-00014.

5. Bauman AE, Reis RS, Sallis JF, Wells JC, Loos RJ, Martin BW. Correlates of physical activity: why are some people physically active and others not? Lancet. 2012; 380(9838):258-271. https://doi.org/10.1016/s0140-6736 (12)60735-1.

6. Dumith SC, Gigante DP, Domingues MR, Kohl HW, 3rd. Physical activity change during adolescence: a systematic review and a pooled analysis. Int J Epidemiol. 2011;40(3):685-698. https://doi.org/10.1093/ije/dyq272.

7. Miljanovic Damjanovic V, Obradovic Salcin L, Zenic N, Foretic N, Liposek S. Identifying Predictors of Changes in Physical Activity Level in Adolescence: A Prospective Analysis in Bosnia and Herzegovina. Int J Environ Res Public Health. 2019;16(14). https://doi.org/10.3390/ijerph 16142573.

8. Štefan L, Sorić M, Devrnja A, Petrić V, MišigojDuraković M. One-year changes in physical activity and sedentary behavior among adolescents: the Croatian Physical Activity in Adolescence Longitudinal Study (CRO-PALS). Int J Adolesc Med Health. 2018;32(5). https://doi.org/10.1515/ijamh-2017-0223.

9. Maric D, Kvesic I, Lujan IK, Bianco A, Zenic N, Separovic V, et al. Parental and Familial Factors Influencing Physical Activity Levels in Early Adolescence: A Prospective Study. Healthcare (Basel). 2020;8(4). https://doi.org/10.3390/ healthcare8040532.

10. Sekulic D, Rodek J, Sattler T. Factors associated with physical activity levels in late adolescence: a prospective study. Med Pr. 2020;71(6):637-647. https://doi.org/10.13075/ mp.5893.01012.

11. Sekulic D, Maric D, Versic S, Zevrnja A, Terzic A, Zenic N. Familial and Parental Predictors of Physical Activity in Late Adolescence: Prospective Analysis over a Two-Year Period. Healthcare (Basel). 2021;9(2). https://doi.org/10. 3390/healthcare9020132.

12. Gilic B, Zenic N, Separovic V, Jurcev Savicevic A, Sekulic D. Evidencing the influence of pre-pandemic sports participation and substance misuse on physical activity during the COVID-19 lockdown: a prospective analysis among older adolescents. Int J Occup Med Environ Health. 2021;34(2): 151-163. https://doi.org/10.13075/ijomeh.1896.01733.

13. Sekulic D, Blazevic M, Gilic B, Kvesic I, Zenic N. Prospective Analysis of Levels and Correlates of Physical
Activity during COVID-19 Pandemic and Imposed Rules of Social Distancing; Gender Specific Study among Adolescents from Southern Croatia. Sustainability. 2020; 12(10):4072. https://doi.org/10.3390/su12104072.

14. Xiao S, Yan Z, Zhao L. Physical Activity, Screen Time, and Mood Disturbance Among Chinese Adolescents During COVID-19. J Psychosoc Nurs Ment Health Serv. 2021;59(4):14-20. https://doi.org/10.3928/02793695-202 01104-04.

15. Moore SA, Faulkner G, Rhodes RE, Brussoni M, ChulakBozzer T, Ferguson LJ, et al. Impact of the COVID-19 virus outbreak on movement and play behaviours of Canadian children and youth: a national survey. Int J Behav Nutr Phys Act. 2020;17(1). https://doi.org/10.1186/ s12966-020-00987-8.

16. Kowalski KC, Crocker PR, Donen RM. The physical activity questionnaire for older children (PAQ-C) and adolescents (PAQ-A) manual. College of Kinesiology, University of Saskatchewan. 2004;87(1):1-38.

17. Benítez-Porres J, Alvero-Cruz JR, Sardinha LB, LópezFernández I, Carnero EA. Cut-off values for classifying active children and adolescentes using the Physical Activity Questionnaire: PAQ-C and PAQ-ACut-off values for classifying active children and adolescents using the Physical Activity Questionnaire: PAQ-C and PAQ-A. Nutr Hosp. 2016;33(5):564.

18. Štveráková T, Jačisko J, Busch A, Šafářová M, Kolář P, Kobesová A. The impact of COVID-19 on Physical Activity of Czech children. PLOS ONE. 2021;16(7):e0254244.

19. Wickel EE, Eisenmann JC. Contribution of youth sport to total daily physical activity among 6- to 12-yr-old boys. Med Sci Sports Exerc. 2007;39(9):1493-500. https://doi. org/10.1249/mss.0b013e318093f56a.

20. Machado-Rodrigues AM, Coelho e Silva MJ, Mota J, Santos RM, Cumming SP, Malina RM. Physical activity and energy expenditure in adolescent male sport participants and nonparticipants aged 13 to 16 years. J Phys Act Health. 2012;9(5):626-33. https://doi.org/10.1123/jpah.9.5. 626.

21. Eime RM, Harvey JT, Charity MJ. Sport drop-out during adolescence: is it real, or an artefact of sampling behaviour? Int J Sport Policy Politics. 2019;11(4):715-726. https://doi.org/10.1080/19406940.2019.1630468.

22. Vaara JP, Vasankari T, Koski HJ, Kyröläinen H. Awareness and Knowledge of Physical Activity Recommendations in Young Adult Men. Front Public Health. 2019;7(310). https://doi.org/10.3389/fpubh.2019.00310.

23. Marques A, Martins J, Santos F, Sarmento H, Carreiro da Costa F. Correlates of school sport participation: A cross-sectional study in urban Portuguese students. 
Science \& Sports. 2014;29(4):e31-e8. https://doi.org/ https://doi.org/10.1016/j.scispo.2013.07.012.

24. Marques A, Martins J, da Costa FC. Scholar sports participation according to age and sex of students from military schools. Br J Sports Med. 2010;44(14):i8. https://doi. org/10.1136/bjsm.2010.078972.24.

25. Gustafson SL, Rhodes RE. Parental Correlates of Physical Activity in Children and Early Adolescents. Sports Med. 2006;36(1):79-97. https://doi.org/10.2165/00007256-200 636010-00006.

26. LeeSM, Nihiser A, Strouse D, Das B, Michael S, Huhman M. Correlates of Children and Parents Being Physically Active Together. J Phys Act Health. 2010;7(6):776. https:// doi.org/10.1123/jpah.7.6.776 10.1123/jpah.7.6.776.

27. Hammed AI, Usman SO, Ezekiel O, Duru JC, Oladimeji OJ, Jirho O. Influence of maternal level of education and socioeconomic status on maternal knowledge of nutrition, physical activity and children's bodyweight of Nigerian school pupils. Exerc Qual Life J. 2021;13(1): 39-44. https://doi.org/10.31382/eqol.210605.

28. Shieh C, Halstead JA. Understanding the impact of health literacy on women's health. J Obstet Gynecol Neonatal Nurs. 2009;38(5):601-610; quiz 10-2. https://doi.org/10. 1111/j.1552-6909.2009.01059.x.

29. Medrano M, Cadenas-Sanchez C, Oses M, Arenaza L, Amasene M, Labayen I. Changes in lifestyle behaviours during the COVID-19 confinement in Spanish children: A longitudinal analysis from the MUGI project. Pediatr Obes. 2021;16(4):e12731. https://doi.org/10.1111/ijpo.12731.

30. Lynch T, Soukup GJ. "Physical education", "health and physical education", "physical literacy" and "health literacy": Global nomenclature confusion. Cogent Educ. 2016; 3(1):1217820. https://doi.org/10.1080/2331186X.2016.12 17820 .

This work is available in Open Access model and licensed under a Creative Commons Attribution-NonCommercial 3.0 Poland License - http://creativecommons.org/licenses/by-nc/3.0/pl/deed.en. 